\title{
Clinical Predictors of Pseudomonas aeruginosa Bacteremia in Emergency Department
}

\author{
Yongsoon Choi, Jin Hui Paik, Ji Hye Kim, Seung Baik Han, and Areum Durey 10 \\ Department of Emergency Medicine, Inha University School of Medicine, Incheon, Republic of Korea \\ Correspondence should be addressed to Areum Durey; areum.durey@gmail.com
}

Received 10 July 2018; Revised 4 September 2018; Accepted 10 September 2018; Published 24 September 2018

Academic Editor: Robert Derlet

Copyright (C) 2018 Yongsoon Choi et al. This is an open access article distributed under the Creative Commons Attribution License, which permits unrestricted use, distribution, and reproduction in any medium, provided the original work is properly cited.

\begin{abstract}
Objectives. Pseudomonas aeruginosa shows higher mortality rate compared to other bacterial infections and is susceptible to a limited number of antimicrobial agents. Considering inadequate empirical treatment of Pseudomonas bacteremia has been associated with increased mortality, it is important for emergency physicians to identify infections by P. aeruginosa. Methods. This was a single-center retrospective case-control study to investigate the clinical predictors of patients diagnosed as Pseudomonas bacteremia in the emergency department (ED) from June 2012 to December 2016. Patients with blood culture positive for Escherichia coli in the same period were chosen as the control group, and type of infection was matched for each patient. Results. A total of 54 cases with Pseudomonas bacteremia and 108 controls with E. coli bacteremia were included. In the case group, $76 \%$ was community-acquired infection, $44 \%$ received inappropriate empirical treatment in the ED, and in-hospital mortality was $30 \%$. Multiple logistic regression showed that respiratory tract infection was an independent risk factor for Pseudomonas bacteremia (OR 6.56, 95\% CI 1.78-23.06; $\mathrm{p}=0.004)$, whereas underlying diabetes mellitus (OR 0.22, 95\% CI 0.07-0.61; $\mathrm{p}=0.004)$ and presentation as urinary tract infection (OR 0.06, 95\% CI 0.02-0.18; $\mathrm{p}<0.001$ ) were negative clinical predictors. Conclusions. We suggest that antipseudomonal antibiotics should be considered beyond simple coverage of Gram-negative bacteria in the ED, especially if the patient is likely to have pneumonia. Having diabetes or presenting with urinary tract infection could be clinical factors unfavorable to use of antipseudomonal antibiotics.
\end{abstract}

\section{Introduction}

Pseudomonas aeruginosa infection shows a higher mortality rate compared to other bacterial infections including Staphylococcus aureus or other Gram-negative bacteria [1] and often occurs in patients with underlying malignancy and immunosuppression [2]. Al-Hasan et al. reported that the 28 -day all-cause mortality of monomicrobial $P$. aeruginosa bacteremia was $25.5 \%$ in a population-based incidence study [3]. Unfortunately, $P$. aeruginosa is susceptible to a limited number of antimicrobial agents suggesting its high mortality could be due to not only inherent virulence, but also delay in administration of appropriate empirical antimicrobial therapy.

It has been documented that inappropriate antimicrobial therapy is associated with adverse outcomes, especially in serious infections caused by Gram-negative bacilli such as $P$. aeruginosa $[4,5]$. Given that emergency physicians treat patients with infections empirically before culture results are available, prompt recognition of Pseudomonas infection and timely initiation of effective antimicrobial therapy are of great importance. Thus, we performed the case-control study to identify the clinical predictors for Pseudomonas bacteremia in the emergency department (ED). In order to determine risk factors, we included a control group consisted of patient with Escherichia coli bacteremia, which is most frequently isolated Gram-negative bacilli from blood.

\section{Methods}

2.1. Study Design. This was a single-center retrospective casecontrol study to investigate the characteristics and risk factors of patients diagnosed as Pseudomonas bacteremia in the ED from June 1, 2012, to December 31, 2016. This study was conducted in a university hospital in Korea which is a tertiary 
hospital with 60,000 patients according to an annual census of ED visits.

2.2. Study Protocol and Population. A 1:2 matched casecontrol study was performed by a blinded observer who was unaware of the clinical outcomes. The records of all positive blood cultures collected at the ED over the 4 years were reviewed from the computer database. Patients were included in the study if their blood cultures were drawn in the ED and the culture results were positive for $P$. aeruginosa or E. coli. Patients with polymicrobial bacteremia were excluded, and only the first bacteremia episodes for each patient were included in the analysis. This review identified 54 adult patients (age, $\geq 18$ years) with clinically significant Pseudomonas bacteremia (defined as the case group). Patients with blood culture positive for E. coli in the same period were chosen as the control group. Type of infection was matched for each patient.

2.3. Variables. The following variables were collected for the case and the control group by reviewing the medical charts: age, sex, comorbidities, predisposing conditions (indwelling urinary catheter, central venous catheter, nasogastric tube, tracheostomy), history of hospitalization or surgery within a year, prior drug exposure including immunosuppressant and chemotherapy within a month, and primary site of infection. Vital signs and results of laboratory tests including neutropenia of enrolled patients were recorded upon ED arrival. Clinical outcome variables such as vasopressor use, intubation, intensive care unit (ICU) admission, and hospital days were also documented.

We investigated antimicrobial susceptibility and multidrug-resistance of the isolated Pseudomonas strains and evaluated appropriateness of the empirical therapy in the ED. In-hospital mortality was the main outcome measurement, yet case patients were followed until one year after bacteremia and 1-year mortality was recorded.

2.4. Definitions. Clinically significant bacteremia was defined as at least one positive blood culture, together with clinical features compatible with systemic inflammatory response syndrome (SIRS) [6]. Pseudomonas bacteremia was diagnosed when at least one of the blood sample cultures was positive for $P$. aeruginosa. Source of bacteremia was determined by two independent doctors based on patients' symptoms, physical examination, and radiological findings. Central venous catheter was defined as all dialysis catheters and medication ports, peripherally inserted central catheters. Neutropenia was defined as a neutrophil count lower than $500 / \mathrm{mm}^{3}$ upon ED arrival. Shock was defined as either systolic blood pressure $<90 \mathrm{mmHg}$ or a requirement for the use of a vasopressor to maintain blood pressure in the ED.

Case patients were classified as hospital-acquired, healthcare-associated, or community-acquired [7]. Infections were considered to be hospital-acquired if the patients were transferred from another hospital after $>48 \mathrm{~h}$ hospitalization. Healthcare-associated infections were defined in accordance with the definitions of Friedman et al. [8]: (1) patients received intravenous therapy, wound care, or specialized nursing care at home; (2) patients attended a hemodialysis clinic in the 30 days before the infection; (3) patients resided in a nursing home or long-term care facility. When patients who did not fall into either criteria, they were categorized as having community-acquired bacteremia.

Appropriateness of Antimicrobial Agents. The initial empirical antimicrobial therapy was considered "appropriate" if the initial antibiotics included at least one antibiotic that was active in vitro antimicrobial susceptibility testing results and when at least the minimum recommended dose was administered via the intravenous route [9]. Otherwise, initial antimicrobial therapy was considered "inappropriate".

Multidrug-Resistance. Among the automated antibiotic susceptibility test results, "intermediate" and "resistance" were categorized as nonsusceptible. Multidrug-resistance (MDR) was defined as Pseudomonas strains resistance to 3 or more of the following eight antipseudomonal agents [10]: $\beta$ lactam/ $\beta$-lactam inhibitors (piperacillin-tazobactam), cephalosporins (ceftazidime, cefepime), carbapenems (imipenem, meropenem), quinolones (ciprofloxacin, levofloxacin), and aminoglycosides (amikacin).

2.5. Microbiology and Antimicrobial Susceptibility. Bloodstream isolates were identified at the species level using the VITEK 2 (bioMérieux Inc., USA). Susceptibility results were interpreted according to Clinical and Laboratory Standards Institute criteria [11].

2.6. Statistical Analyses. Data with a normal distribution were expressed as mean \pm standard deviation and were analyzed by independent samples $t$ test. Data with a skewed distribution were expressed as medians and interquartile ranges and were analyzed by Mann-Whitney $U$ test. Categorical variables were compared using $\chi^{2}$ test or Fisher exact test depending on the sample size. Univariate analysis followed by multivariable logistic regression analysis was performed in this case-control study to identify independent risk factors. Variables with a $p$ value of $<0.10$ in the univariate analysis were candidates for multivariate analysis using a backward elimination method. A $p$ value $<0.05$ was considered statistically significant. All statistical analyses were performed using Medcalc for Windows, version 17.6 (MedCalc software, Ostend, Belgium).

\section{Results}

3.1. Study Population and Clinical Characteristics. Table 1 shows clinical characteristics of study population. During the study period, a total of 54 patients with Pseudomonas bacteremia were identified and included in the analysis as case group. The most common underlying diseases were malignancy (solid tumor and hematologic malignancy, 55\%). As shown in Table 2, community-acquired type was most prevalent (76\%) among patients with Pseudomonas bacteremia, next to hospital-acquired (17\%) and healthcareassociated (7\%) regarding type of infection. A total of 108 
TABLE 1: Comparison of clinical characteristics of 54 case patients with Pseudomonas bacteremia and of 108 control patients with E. coli bacteremia in the ED.

\begin{tabular}{|c|c|c|c|}
\hline Characteristics & Case $(\mathrm{n}=54)$ & Control $(\mathrm{n}=108)$ & $\mathrm{P}$ value \\
\hline Age & $66.4 \pm 15.2$ & $73.5(71.0-76.0)$ & $0.021^{*}$ \\
\hline Male, no. (\%) & $28(52)$ & $30(28)$ & $0.002^{* *}$ \\
\hline \multicolumn{4}{|l|}{ Comorbid conditions, no. (\%) } \\
\hline Diabetes mellitus & $11(20)$ & $47(44)$ & $0.003^{* *}$ \\
\hline Cardiovascular disease & $10(19)$ & $24(22)$ & 0.586 \\
\hline Respiratory disease & $1(2)$ & $7(7)$ & 0.201 \\
\hline Chronic renal failure & $7(13)$ & $14(13)$ & 1.0 \\
\hline Liver cirrhosis & $3(6)$ & $5(5)$ & 1.0 \\
\hline Rheumatologic disease & $1(2)$ & $8(7)$ & 0.274 \\
\hline Neurodegenerative disease & $9(17)$ & $27(25)$ & 0.230 \\
\hline Solid tumor & $25(46)$ & $26(24)$ & $0.004^{* *}$ \\
\hline Hematological malignancy & $5(9)$ & $1(1)$ & $0.016^{*}$ \\
\hline \multicolumn{4}{|l|}{ Predisposing conditions, no. (\%) } \\
\hline Indwelling urinary catheter & $3(6)$ & $10(9)$ & 0.546 \\
\hline Presence of central venous catheter & $4(7)$ & $3(3)$ & 0.223 \\
\hline Nasogastric tube in place & $2(4)$ & $2(2)$ & 0.601 \\
\hline Tracheostomy & $1(2)$ & $2(2)$ & 1.0 \\
\hline Prior hospitalization within 1 year, no. (\%) & $40(74)$ & $52(48)$ & $0.001^{* *}$ \\
\hline Prior surgery within 1 year, no. (\%) & $13(24)$ & $10(9)$ & $0.011^{*}$ \\
\hline \multicolumn{4}{|l|}{ Drug exposure within 1 month, no. (\%) } \\
\hline Immunosuppressant & $2(4)$ & $7(6)$ & 0.718 \\
\hline Chemotherapy & $17(31)$ & $4(4)$ & $<0.0001^{* * *}$ \\
\hline \multicolumn{4}{|l|}{ Source of bacteremia } \\
\hline Respiratory tract & $21(39)$ & $5(5)$ & $<0.0001^{* * *}$ \\
\hline Gastrointestinal tract & $17(31)$ & $23(21)$ & 0.157 \\
\hline Urinary tract & $8(15)$ & $84(78)$ & $<0.0001^{* * *}$ \\
\hline Skin and soft tissue & $5(9)$ & 0 & $0.003^{* *}$ \\
\hline Central venous catheter & 0 & 0 & \\
\hline Unknown & $4(7)$ & 0 & $0.011^{*}$ \\
\hline \multicolumn{4}{|l|}{ Vital signs on presentation } \\
\hline SBP, mm Hg & $117.4 \pm 29.3$ & $125.7 \pm 24.7$ & 0.061 \\
\hline DBP, mm Hg & $70.8 \pm 16.3$ & $72.6 \pm 15.6$ & 0.514 \\
\hline $\mathrm{PR}$, beats/min & $104.4 \pm 22.2$ & $100(94-104)$ & 0.409 \\
\hline $\mathrm{RR}$, breaths/min & $19(18.0-21.3)$ & $20(18-20)$ & 0.820 \\
\hline Body temperature, ${ }^{\circ} \mathrm{C}$ & $37.8 \pm 1.2$ & $38.4(38.1-38.8)$ & $0.025^{*}$ \\
\hline Saturation, $\%$ & $97(95-98)$ & $96(96-97)$ & 0.436 \\
\hline \multicolumn{4}{|l|}{ Laboratory findings on presentation } \\
\hline Leukocyte count, $\mathrm{x} 10^{9}$ cells $/ \mathrm{mL}$ & $8810(6729-12623)$ & 10475 (9303-11099) & 0.223 \\
\hline Neutropenia & $15(28)$ & 0 & $<0.0001^{* * *}$ \\
\hline Hemoglobin, g/dL & $10.4 \pm 2.3$ & $11.6 \pm 1.7$ & $<0.001^{* * *}$ \\
\hline Platelet, $\times 10^{3} / \mu 1$ & $178.0(141.9-213.6)$ & $181(163-197)$ & 0.816 \\
\hline $\mathrm{CRP}, \mathrm{mg} / \mathrm{dL}$ & $10.46(7.87-17.53)$ & $9.64(7.79-11.5)$ & 0.464 \\
\hline Lactic acid, mmol/L & $2.25(1.43-4.30)$ & $2.50(2.19-2.90)$ & 0.642 \\
\hline Arterial blood gas $\mathrm{pH}$ & $7.44(7.40-7.46)$ & $7.44(7.43-7.46)$ & 0.310 \\
\hline $\mathrm{PCO}_{2}, \mathrm{~mm} \mathrm{Hg}$ & $28.9(25.8-31.1)$ & $29.8(28.5-30.8)$ & 0.252 \\
\hline $\mathrm{PO}_{2}, \mathrm{~mm} \mathrm{Hg}$ & $77.1(69.8-83.9)$ & $75(72.3-77.9)$ & 0.338 \\
\hline $\mathrm{HCO}_{3}{ }^{-}, \mathrm{mmol} / \mathrm{L}$ & $18.2 \pm 6.2$ & $21(20.1-21.8)$ & $0.036^{*}$ \\
\hline $\mathrm{SpO}_{2}, \mathrm{~mm} \mathrm{Hg}$ & $95.7(95.3-97.0)$ & $95.6(95.0-96.3)$ & 0.572 \\
\hline Presentation with shock, no. (\%) & $20(37)$ & $26(24)$ & 0.063 \\
\hline Use of vasopressor, no. (\%) & $20(37)$ & $25(23)$ & 0.063 \\
\hline Intubation, no. (\%) & $14(26)$ & $10(9)$ & $0.005^{* *}$ \\
\hline
\end{tabular}


TABLE 1: Continued.

\begin{tabular}{lccc}
\hline Characteristics & Case $(\mathrm{n}=54)$ & Control $(\mathrm{n}=108)$ & P value \\
\hline ICU care, no. $(\%)$ & $24(44)$ & $28(26)$ & $0.017^{*}$ \\
Hospital days & $9(3-15)$ & $8(6-9)$ & 0.980 \\
In-hospital death, no $(\%)$ & $16(30)$ & $9(8)$ & $<0.001^{* * *}$ \\
\hline
\end{tabular}

${ }^{*} \mathrm{p}<0.05,{ }^{* *} \mathrm{p}<0.01{ }^{* * *} \mathrm{p}<0.001$ : significant change from baseline values.

TAble 2: Additional characteristics of patients with Pseudomonas bacteremia in the ED including antimicrobial susceptibility profile of the isolates.

\begin{tabular}{lc}
\hline Characteristics & Case $(\mathrm{n}=54)$ \\
\hline Infection type & \\
Community-acquired & $76 \%$ \\
Healthcare-associated & $7 \%$ \\
Hospital-acquired & $17 \%$ \\
\hline Antimicrobial susceptibility & \\
Aztreonam & $50 \%$ \\
Ceftazidime & $85 \%$ \\
Cefepime & $83 \%$ \\
Piperacillin-Tazobactam & $81 \%$ \\
Ciprofloxacin & $80 \%$ \\
Amikacin & $96 \%$ \\
Imipenem & $83 \%$ \\
Meropenem & $83 \%$ \\
Colistin & $100 \%$ \\
\hline Multidrug-resistance & $22 \%$ \\
Use of inappropriate antibiotics & $44 \%$ \\
1-year mortality, no (\%) & $33(61)$ \\
\hline
\end{tabular}

patients with $E$. coli bacteremia matched for type of infection during the period were compared to case patients.

Case patients were more often male ( $52 \%$ versus $28 \%$; $\mathrm{p}=0.002)$ and younger than control patients (66.4 versus 73.5 years; $\mathrm{p}=0.021)$. As for comorbid conditions, solid tumor $(46 \%$ versus $24 \% ; \mathrm{p}=0.004)$ and hematological malignancy ( $9 \%$ versus $1 \% ; \mathrm{p}=0.016$ ) were more prevalent in the Pseudomonas group. In contrast, diabetes mellitus $(20 \%$ versus $44 \%$; $\mathrm{p}=0.003$ ) was more prevalent in the $E$. coli group. In the case group, history of prior hospitalization and surgery within 1 year was more frequently observed. Moreover, $31 \%$ of the case group received chemotherapy within 1 month compared to $4 \%$ of the control group ( $\mathrm{p}<0.0001)$.

When the sources of bacteremia were evaluated, respiratory tract $(39 \%$ versus $5 \%$; $<<0.0001)$, skin and soft tissue ( $9 \%$ versus $0 \% ; \mathrm{p}=0.003)$, and unknown primary site $(7 \%$ versus $0 \% ; \mathrm{p}=0.011)$ were more frequent in the Pseudomonas group, yet urinary tract infection (15\% versus $78 \%$; $\mathrm{p}<0.0001$ ) was more significantly common in the E. coli group. Upon ED arrival, case group presented with less higher body temperature and lower level of hemoglobin and HCO3compared to the control group. In addition, presentation with neutropenia was more prevalent in the Pseudomonas group (28\% versus $0 \%$; $<<0.0001)$.
3.2. Treatment Outcomes. Table 1 shows that case patients were more frequently intubated in the ED (26\% versus $9 \%$; $\mathrm{p}=0.005)$ and ICU care was more common compared to the control patients ( $44 \%$ versus $26 \%$; $\mathrm{p}=0.17$ ). The in-hospital mortality was significantly higher in the Pseudomonas group than the E. coli group (30\% versus $8 \%, \mathrm{p}<0.001$ ).

3.3. Clinical Predictors for Pseudomonas Bacteremia. As shown in Table 3, being male, underlying solid tumor, history of hospitalization, or surgery within a year was associated with Pseudomonas bacteremia in the ED by simple logistic regression, yet was not statistically significant in multiple logistic regression analysis. Notably, odds ratio of hematologic malignancy, history of chemotherapy within a month, and presence of neutropenia upon ER visit were 10.91 (95\% CI 1.24-95.96; $\mathrm{p}=0.031$ ), 11.94 (95\% CI 3.77-37.8; $\mathrm{p}<0.001$ ), and 41.1 (95\% CI 5.25-321.99; p < 0.0001), respectively.

Multiple logistic regression showed that respiratory tract infection was an independent risk factor for Pseudomonas bacteremia in the ED (OR 6.56, 95\% CI 1.78-23.06; $\mathrm{p}=$ 0.004), whereas age (OR 0.96, 95\% CI 0.92-0.99; $\mathrm{p}=0.029$ ), underlying diabetes mellitus (OR 0.22, 95\% CI 0.07-0.61; p = 0.004 ), and presentation as urinary tract infection (OR 0.06, 95\% CI 0.02-0.18; $\mathrm{p}<0.001)$ were negative clinical predictors for Pseudomonas bacteremia.

3.4. Antimicrobial Susceptibility of Isolated P. aeruginosa. Of the 54 strains of $P$. aeruginosa evaluated, the aztreonam showed the lowest susceptibility rate (50\%) among antibiotics tested (Table 2). Susceptibility rates of other antibiotics ranged from $80 \%$ to $96 \%$. Carbapenems and colistin were effective against $83 \%$ and $100 \%$ of the isolates. MDR strains (resistant to $\geq 3$ antibiotic groups) were identified in 12 isolates (22\%).

The choice in empirical antibiotics was made by the emergency physician in charge of the patient. Notably, among the patients with Pseudomonas bacteremia, 44\% (24 out of 54) received inappropriate antimicrobial therapy in the ED. During one-year follow-up after bacteremia, 33 out of 54 case patients died resulting in 1-year all-cause mortality of $61 \%$.

\section{Discussion}

$P$. aeruginosa causes infections that usually occur late during hospital stay [12]. Affected patients are often hospitalized in an ICU, are immunocompromised, and have multiple invasive devices $[13,14]$. Schechner et al. reported that independent predictors of Pseudomonas bacteremia were severe immunodeficiency, age $>90$ years, antimicrobial therapy 
TABLE 3: Logistic regression analysis of clinical predictors of patients for P. aeruginosa bacteremia in the ED.

\begin{tabular}{|c|c|c|c|c|}
\hline \multirow{2}{*}{ Characteristics } & \multicolumn{2}{|c|}{ Simple logistic analysis } & \multicolumn{2}{|c|}{ Multiple logistic analysis } \\
\hline & OR $(95 \% \mathrm{CI})$ & $\mathrm{P}$ value & OR $(95 \% \mathrm{CI})$ & $\mathrm{P}$ value \\
\hline Age & & & $0.9(0.92-0.99)$ & $0.029^{*}$ \\
\hline Male, no. (\%) & $2.8(1.41-5.52)$ & $0.003^{* *}$ & & \\
\hline \multicolumn{5}{|l|}{ Comorbid conditions, no. (\%) } \\
\hline Diabetes mellitus & & & $0.2(0.07-0.61)$ & $0.004^{* *}$ \\
\hline Solid tumor & $2.7(1.35-5.43)$ & $0.004^{* *}$ & & \\
\hline Hematologic malignancy & $10.9(1.24-95.96)$ & $0.031^{*}$ & & \\
\hline Prior hospitalization within 1 year, no. (\%) & $3.1(1.50-6.29)$ & $0.002^{* *}$ & & \\
\hline Prior surgery within 1 year, no. (\%) & $3.1(1.26-7.65)$ & $0.013^{*}$ & & \\
\hline \multicolumn{5}{|l|}{ Drug exposure within 1 month, no. (\%) } \\
\hline Chemotherapy & $11.9(3.77-37.80)$ & $<0.001^{* * *}$ & & \\
\hline Neutropenia & $41.1(5.25-321.99)$ & $<0.001^{* * *}$ & & \\
\hline \multicolumn{5}{|l|}{ Primary site of infection } \\
\hline Respiratory tract & & & $6.5(1.78-24.06)$ & $0.004^{* *}$ \\
\hline Urinary tract & & & $0.06(0.02-0.18)$ & $<0.001^{* * *}$ \\
\hline
\end{tabular}

${ }^{*} \mathrm{p}<0.05,{ }^{* *} \mathrm{p}<0.01{ }^{* * *} \mathrm{p}<0.001$ : significant change from baseline values.

$\mathrm{CI}=$ confidence interval; $\mathrm{OR}=$ odds ratio.

within 30 days, and presence of a central venous catheter or urinary device [15]. In contrast to these previous reports focusing on all cases of Pseudomonas bacteremia, we limited study population to cases presented to the ED. According to our study, $76 \%$ of Pseudomonas bacteremia presented to the ED over the 4 years was community-acquired suggesting that possibility of $P$. aeruginosa infection should not be overlooked in the community setting.

Although MDR Pseudomonas infections have been increasing since 2000s [16], susceptibility rates of the isolated P. aeruginosa in our study ranged from $80 \%$ to $100 \%$ except for aztreonam with a susceptibility rate of $50 \%$, and $22 \%$ of them revealed MDR. Despite this favorable profile of susceptibility, 24 out of 54 case patients (44\%) still received inappropriate empirical treatment in the $\mathrm{ED}$, which implies low suspicion could be a matter regarding treatment of Pseudomonas bacteremia in the ED rather than MDR $P$. aeruginosa.

In the present study, in-hospital mortality was 30\% in line with previous observations [17], yet 1-year mortality was higher [3] probably due to high prevalence of cancer in the study population. In several studies, inadequate empirical treatment of Pseudomonas bacteremia has been associated with increased mortality $[2,18]$. Lodise et al. who found that delaying appropriate therapy for approximately 2 days significantly increases the risk of mortality in patients with Pseudomonas bloodstream infection [19]. However, overuse of antipseudomonal agents due to concern of delaying treatment could increase resistance rates leading to limit future options [20]. Therefore, it is very important for emergency physicians to identify patients who are at risk of developing Pseudomonas infection and use appropriate empirical therapy in the ED.

In our study, we presented our recent experiences on Pseudomonas bacteremia in the ED, described its clinical characteristics, and tried to identify potential risk factors of
Pseudomonas bacteremia by comparing patients with E. coli bacteremia. We chose E. coli bacteremia as the control group in order to narrow clinical scenario into when sepsis caused by Gram-negative bacteria is suspected in the ED. Because antipseudomonal antibiotics already include coverage of common Gram-negative bacteria such as E. coli, we expect our results could help emergency physicians select empirical antibiotics properly in the ED, for example, ceftazidime over cefotaxime or piperacillin-tazobactam over ampicillinsulbactam. Our results showed that respiratory tract infection was an independent risk factor for Pseudomonas bacteremia in the ED, which was consistent with the recent findings of Hammer KL et al. [21].

\section{Limitations}

The main limitation of the present study is that it was a singlecenter retrospective study and the number of case patients with Pseudomonas bacteremia was small. Also, we only included clinically significant bacteremia which was defined as positive blood culture with SIRS, and prior antibiotic use was not assessed as a risk factor for Pseudomonas bacteremia.

Therefore, additional factors related to Pseudomonas bacteremia may not be revealed. In addition, our study did not refer to all patients presenting to the ED with bacteremia, but only patients with documented Gram-negative bacteremia.

\section{Conclusions}

In conclusion, $P$. aeruginosa is an important pathogen in communities as well as in hospitals. The mortality rate of Pseudomonas bacteremia was significantly high compared to that of E. coli bacteremia in our study; thus higher degree of suspicion in the ED is required to provide appropriate antimicrobial therapy. We suggest that antipseudomonal 
antibiotics should be considered beyond simple coverage of Gram-negative bacteria in the ED, especially if the patient is likely to have pneumonia. Having diabetes or presenting with urinary tract infection could be clinical factors unfavorable to use of antipseudomonal antibiotics.

\section{Data Availability}

The data used to support the findings of this study are available from the corresponding author upon request.

\section{Conflicts of Interest}

The authors of this study have no conflicts of interest to report.

\section{Acknowledgments}

This work was supported by Inha University Hospital Research Grant.

\section{References}

[1] J. T. Thaden, L. P. Park, S. A. Maskarinec, F. Ruffin, V. G. Fowler, and D. van Duin, "Results from a 13-Year Prospective Cohort Study Show Increased Mortality Associated with Bloodstream Infections Caused by Pseudomonas aeruginosa Compared to Other Bacteria," Antimicrobial Agents and Chemotherapy, vol. 61, no. 6, 2017.

[2] C. I. Kang, S. H. Kim, H. B. Kim et al., "Pseudomonas aeruginosa bacteremia: Risk factors for mortality and influence of delayed receipt of effective antimicrobial therapy on clinical outcome," Clinical Infectious Diseases, vol. 37, no. 6, pp. 745-751, 2003.

[3] M. N. Al-Hasan, J. W. Wilson, B. D. Lahr, J. E. Eckel-Passow, and L. M. Baddour, "Incidence of Pseudomonas aeruginosa Bacteremia: A Population-Based Study," American Journal of Medicine, vol. 121, no. 8, pp. 702-708, 2008.

[4] S. T. Micek, A. E. Lloyd, D. J. Ritchie, R. M. Reichley, V. J. Fraser, and M. H. Kollef, "Pseudomonas aeruginosa bloodstream infection: importance of appropriate initial antimicrobial treatment," Antimicrobial Agents and Chemotherapy, vol. 49, no. 4, pp. 13061311, 2005.

[5] C.-I. Kang, S.-H. Kim, W. B. Park et al., "Bloodstream infections caused by antibiotic-resistant gram-negative bacilli: risk factors for mortality and impact of inappropriate initial antimicrobial therapy on outcome," Antimicrobial Agents and Chemotherapy, vol. 49, no. 2, pp. 760-766, 2005.

[6] M. M. Levy, M. P. Fink, J. C. Marshall et al., "2001 SCCM/ESICM/ACCP/ATS/SIS international sepsis definitions conference," Intensive Care Medicine, vol. 29, no. 4, pp. 530-538, 2003.

[7] H. Lee, S. B. Han, J. H. Kim, S. Kang, and A. Durey, "Risk factors of urinary tract infection caused by extended spectrum $\beta$-lactamase-producing Escherichia coli in emergency department," The American Journal of Emergency Medicine, 2018.

[8] N. D. Friedman, K. S. Kaye, J. E. Stout et al., "Health care-associated bloodstream infections in adults: a reason to change the accepted definition of community-acquired infections," Annals of Internal Medicine, vol. 137, no. 10, pp. 791-797, 2002.
[9] S. E. Cain, J. Kohn, P. B. Bookstaver, H. Albrecht, and M. N. Al-Has, "Stratification of the impact of inappropriate empirical antimicrobial therapy for gram-negative bloodstream infections by predicted prognosis," Antimicrobial Agents and Chemotherapy, vol. 59, no. 1, pp. 245-250, 2015.

[10] A.-P. Magiorakos, A. Srinivasan, R. B. Carey et al., "Multidrugresistant, extensively drug-resistant and pandrug-resistant bacteria: an international expert proposal for interim standard definitions for acquired resistance," Clinical Microbiology and Infection, vol. 18, no. 3, pp. 268-281, 2012.

[11] Clinical and Laboratory Standards Institute, Performance Standards for Antimicrobial Susceptibility Testing; Seventeenth Information Supplement, CLSI document M100-S17, Clinical and Laboratory Standards Institute, Wayne, Pa, USA, 2011.

[12] R. Gaynes and J. R. Edwards, "Overview of nosocomial infections caused by gram-negative bacilli," Clinical Infectious Diseases, vol. 41, no. 6, pp. 848-854, 2005.

[13] G. Pier and R. Ramphal, "Pseudomonas aeruginosa," in Mandell, Douglas, and Bennett's principles and practice of infectious diseases, G. L. Mandell, J. E. Bennett, and R. Dolin, Eds., pp. 2587-2615, Elsevier/Churchill Livingstone, Philadelphia, USA, 6 edition, 2005.

[14] M. E. Falagas and P. Kopterides, "Risk factors for the isolation of multi-drug-resistant Acinetobacter baumannii and Pseudomonas aeruginosa: a systematic review of the literature," Journal of Hospital Infection, vol. 64, no. 1, pp. 7-15, 2006.

[15] V. Schechner, V. Nobre, K. S. Kaye et al., "Gram-negative bacteremia upon hospital admission: When should Pseudomonas aeruginosa be suspected?" Clinical Infectious Diseases, vol. 48, no. 5, pp. 580-586, 2009.

[16] C. Cattaneo, S. Casari, F. Bracchi et al., "Recent increase in enterococci, viridans streptococci, Pseudomonas spp. and multiresistant strains among haematological patients, with a negative impact on outcome. Results of a 3-year surveillance study at a single institution," Infectious Diseases, vol. 42, no. 5, pp. 324-332, 2010.

[17] V. Aloush, S. Navon-Venezia, Y. Seigman-Igra, S. Cabili, and Y. C. Carmeli, "Multidrug-resistant Pseudomonas aeruginosa: risk factors and clinical impact," Antimicrobial Agents and Chemotherapy, vol. 50, no. 1, pp. 43-48, 2006.

[18] G. P. Bodey, L. Jadeja, and L. Elting, "Pseudomonas Bacteremia: Retrospective Analysis of 410 Episodes," JAMA Internal Medicine, vol. 145, no. 9, pp. 1621-1629, 1985.

[19] T. P. Lodise Jr., N. Patel, A. Kwa et al., "Predictors of 30day mortality among patients with Pseudomonas aeruginosa bloodstream infections: Impact of delayed appropriate antibiotic selection," Antimicrobial Agents and Chemotherapy, vol. 51, no. 10 , pp. 3510-3515, 2007.

[20] C.-I. Kang, S.-H. Kim, W. B. Park et al., "Risk factors for antimicrobial resistance and influence of resistance on mortality in patients with bloodstream infection caused by Pseudomonas aeruginosa," Microbial Drug Resistance, vol. 11, no. 1, pp. 68-74, 2005.

[21] K. L. Hammer, J. A. Justo, P. B. Bookstaver, J. Kohn, H. Albrecht, and M. N. Al-Hasan, "Differential effect of prior $\beta$-lactams and fluoroquinolones on risk of bloodstream infections secondary to Pseudomonas aeruginosa," Diagnostic Microbiology and Infectious Disease, vol. 87, no. 1, pp. 87-91, 2017. 


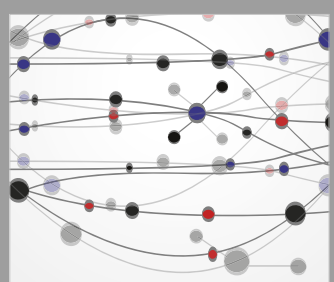

The Scientific World Journal
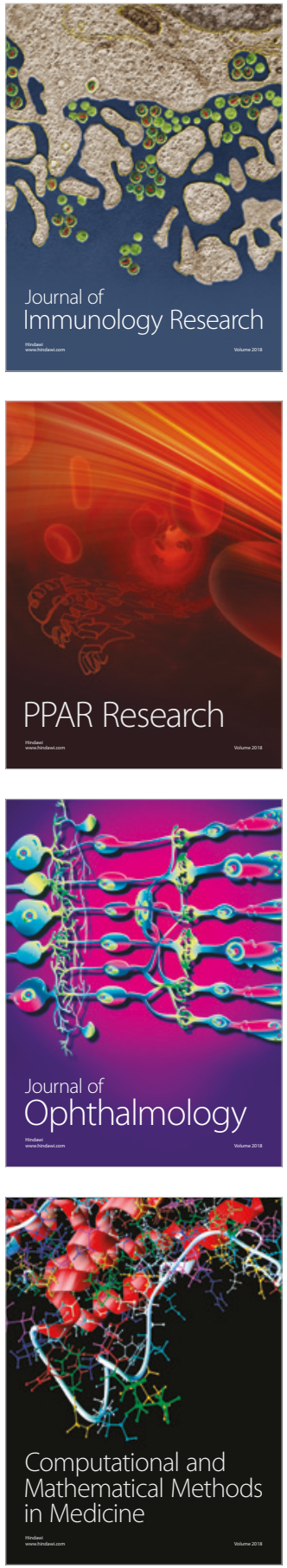

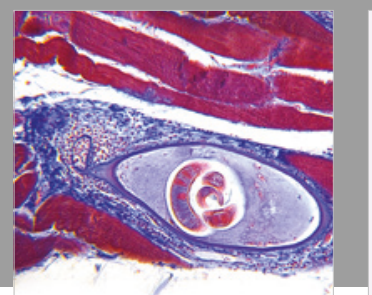

Gastroenterology Research and Practice

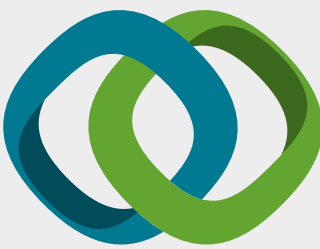

\section{Hindawi}

Submit your manuscripts at

www.hindawi.com
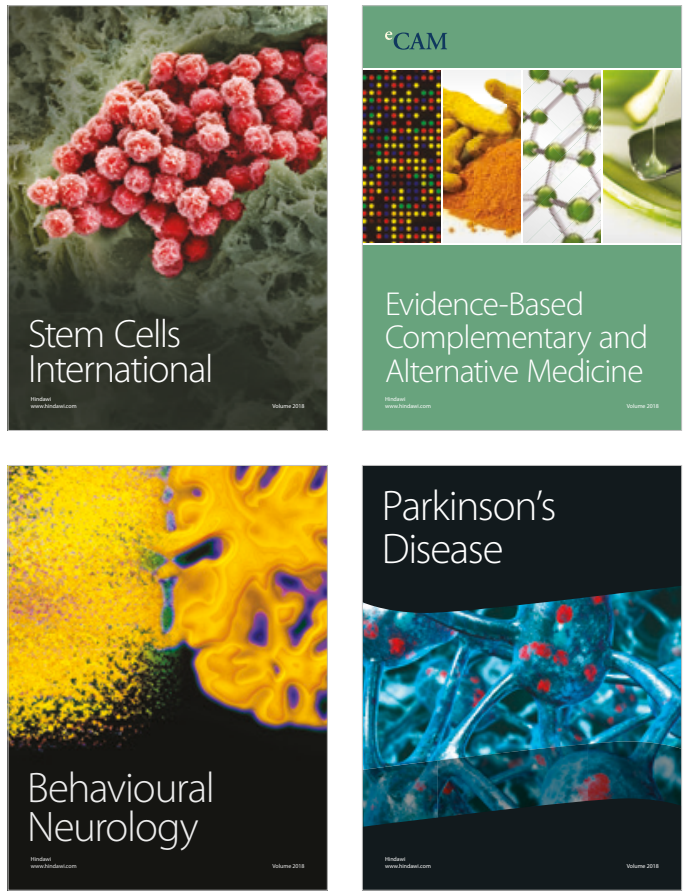

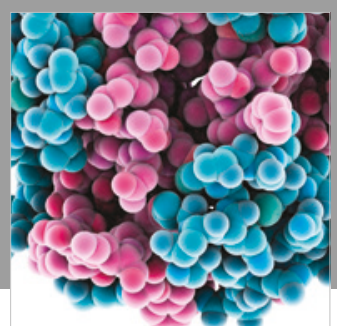

ournal of

Diabetes Research

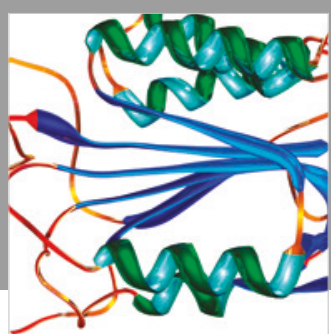

Disease Markers
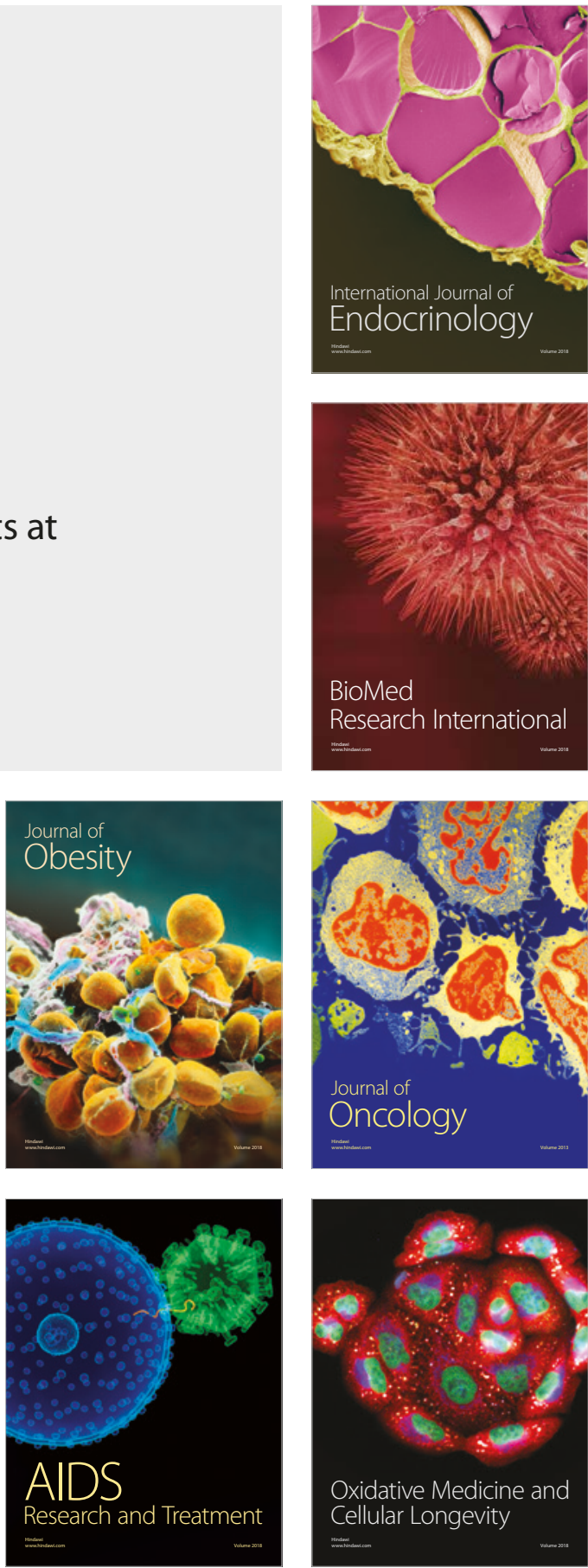